\title{
Discovery of Helical Magnetic Fields in AGN, and CME and Ejection of Large-Scale Magnetic Fields from AGN
}

\author{
Han-Shu Chu \\ Purple Mountain Observatory, China Academy of Sciences, Nanjing \\ 210008, China
}

L. B. Baath

Center for Imaging Technologies, University of Halmstad, S-301 18

Halmstad, Sweden

Fu-Jun Zhang

Shanghai Observatory, China Academy of Sciences, Shanghai, China

\author{
R. E. Spencer
}

University of Manchester, N.R.A.L., Jodrell Bank, UK

\begin{abstract}
Magnetic Fields are the crucial and most important ingredient involved in the processes of various violent activities in Active Galactic Nuclei and other celestial bodies. The generally accepted 2-sided symmetric-jets model of active galactic nuclei (AGN) does not include the magnetic fields. We present here the first direct detection of helical magnetic field in AGN, and the first direct detection of ejection of large scale magnetic fields from AGN. (CME) 2. The annular (helical) magnetic field is responsible for the collimation of the jet (through Pinch Effect) and may be of crucial importance for extraction of black hole rotational energy. 3. The CMEs are responsible for sporadic ejection of jet components in AGN, while the general accepted 2-sided jets pertain to the quiescent Jets. 4.Observations show that the CME with Asymmetric Jets can explain many phenomena and much of the morphological diversities in AGN.
\end{abstract}

\section{Introduction}

High resolution imaging available in radio astronomy Very Long Baseline Interferometry (VLBI) may help to unveil some of the invisible in other wavelengths important details of the High Energy Processes and Phenomena in Astrophysics. The author present here some of the recent findings which seem of importance to the understanding of the nature of AGN. The orthodox model of AGN, as is generally accepted, is that of Rees. We have found an AGN (3C 147)which has a counter-jet much longer than the main jet. It also has an unusual sideways ejection from the nuclear region. Both of these and the 3-jet structure per se 


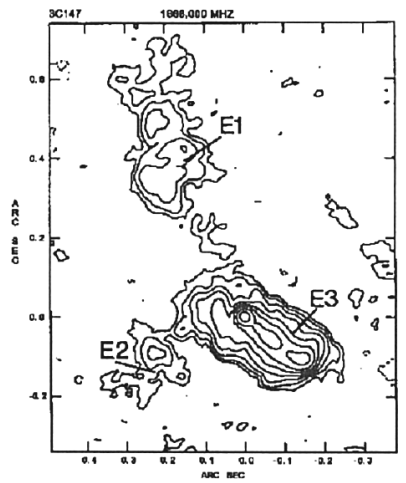

Figure 1. EVN-MERLIN combined map of 3C147 (Zhang et al. 1991)

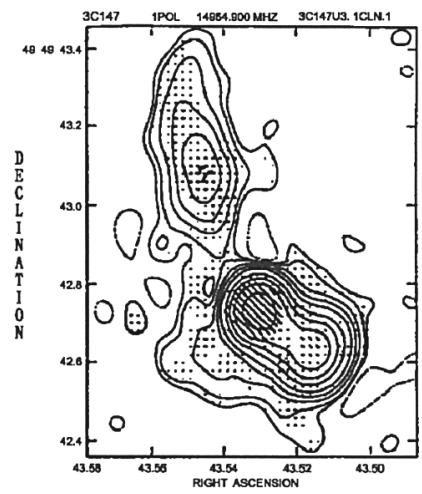

Figure 2. VLA map at $15 \mathrm{GHz}$ from 1983 data (Kellermann and Crane, unpublished).Polarization line $1 "=0.379 \mathrm{Jy} /$ beam; contours at $100,50,20,10,5,2,1,0.5,0.2,0.1,-0.1$ percent of the peak brightness of 1.41 Jy per beam. Synthesized beam 0.0910.082" P.A.-13.6".

do not seem to conform with the general accepted model, and are also not explained by relativistic beaming effects. We speculate that this is due to coronal mass ejection (CME) in the accretion disk corona. Recently, we have found convincing new evidence that this is true. We have also found direct evidence of helical magnetic fields in the core region of $3 \mathrm{C} 147$. These results may be of importance to the understanding of AGN.

2. Finding of An Unusual 3-Jet Structure in 3C147, Revealing Helical Magnetic Fields and New Unknown Ejection Mechanism in AGN

An unusual 3-jet structure was found and confirmed in QSR 3C147 on the basis of combined EVN-MERLIN VLBI mapping, in conjunction with multi-frequency 
VLBI studies and VLA Observations in intensity and polarization at many frequencies (van Breugel, Miley, \& Heckman 1984 Zhang et al. 1991; Chu et al. 1992; Chu et al. 1998). The figures in these papers show a bright main jet 230 mas (2pc) long to the SW enveloping the core and a much longer ( 700 mas) and less bright jet to the $\mathrm{N}$. A third extended component ( 50 mas long) exists to the SE of the core as shown most clearly by the EVN-MERLIN image of Zhang et al. (1991, fig. 1). This component is a previously unknown off-axis ejection from the nuclear region and is invisible in optical, IR, X-ray and other wavelengths. (by present technology). This 3-jet structure is important: it is un-expected in the 2-sided symmetric-jets model and is in contradiction to the generally accepted relativistic beaming model. It also leads to the new findings of helical magnetic fields and intrinsically asymmetric jets in AGN and to the new findings of CME and ejection of large scale magnetic fields from AGN.

\section{Helical Magnetic Fields and CME and Ejection of Large Scale Magnetic Fields}

As shown in Figs. 1 and 2, the main jet of 3C147 is surrounded by a cocoon of extended emission. The jet is also edge brightened in our EVN along map (Zhang et al. 1991). These features, together with the high degree of collimation, can be explained if there is an ordered annular (helical) magnetic field in the core region and jet. And the author was able to find the VLA maps of Kellermann \& Crane (Fig. 2) and van Brengel et al. (Fig. 4) indeed show strong linear polarization near the core of $3 \mathrm{C} 147$, suggesting the presence of helical magnetic field in the core region and jet of the source. Magnetic Pinch Effects of such a field will keep the jet matter flowing in the axial direction well collimated. This in turn indicates that the helical magnetic field and its associated Pinch Effects are responsible for the collimation of the jet. The detection of a helical magnetic field in an AGN indicates also that the 2-sided jets are intrinsically asymmetrical and independent, since magnetic fields in the 2-sides of the accretion disk are intrinsically asymmetrical. It is also interesting to point out that this annular (circular) magnetic field, its existence and important significance, were disclosed behind the directly perceived "bushy" Linear polarization E vectors in the map of $3 \mathrm{C} 147$.

The annular (helical) magnetic field may also be of importance for extraction of black hole rotational energy. The much longer length of the northern jet than the main jet is quiet unusual. We suggest that these two jets do not constitute a twin jet since the brightness and length ratios do not fit the standard relativistic beaming model (as according to the standard relativistic beaming model, the counter-jet should be seen much foreshortened and much diminished in brightness).

The $\mathrm{N}$ jet shows also no connection with the core on the combined EVNMERLIN map, only a generic relation to the cocoon surrounding the main jet. The MERLIN and VLA maps also show that the $\mathrm{N}$ jet is isolated from the core. These indicate that the $\mathrm{N}$ jet may not be formed by a continuous flow from the core but possibly due to an outburst (or CME) from an accretion disk corona. Its diffuse, fragmented and interrupted structure in our combined $6 \mathrm{~cm}$ map 


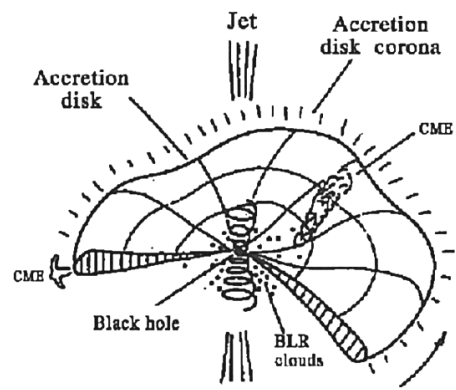

Figure 3. A model of jet origin, BLR clouds and activity in 3C147. The accretion disk may be warped. The two jets may be asymmetric and independent. The counter-jet may not be seen due to Doppler beaming.

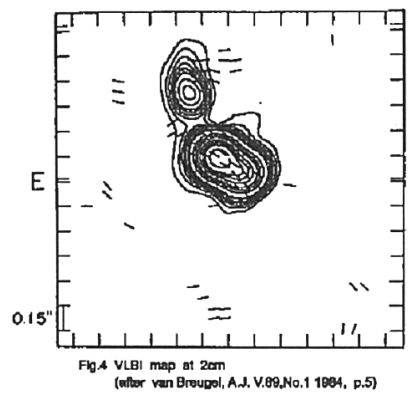

Figure 4. VLBI map at 2cm (after van Breugel et al. 1984)

(Zhang et al. 1991) is more consistent with this hypothesis than its origin as a jet from a double black hole.

The most unusual feature is the sideways ejection, totally unexpected in the generally accepted 2-sided jet model. What is the origin of this unusual feature? We think the non-axial character of the ejection itself may afford a clue to guide the research, and to give an insight into the nature of this strange QSR 3C147. And we proposed an accretion disk corona model (Chu et al. 1992) to account for the origin of this (Fig. 3)

The sideways ejection is non-axial, this reveals that a previous unknown non-axial ejection mechanism may be operating in AGN, along with that giving the main jet.

The accretion disk is very likely to form a corona somewhat similar to that on the sun and in binary X-ray sources. Since there are basic qualitative similarities between the creation of solar and galactic magnetic fields by a MHD dynamo process (Parker 1990), we can expect such features as prominences and flares also to occur in a galactic disk corona. The non-axial ejection could thus be of magnetic origin. 


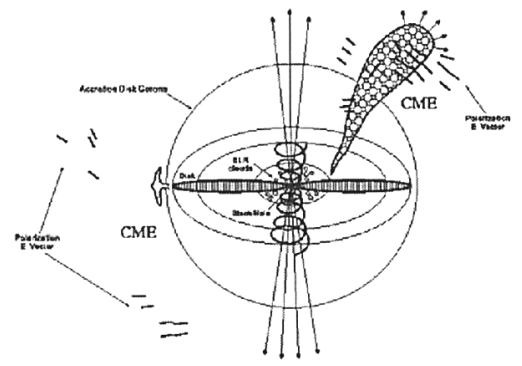

Figure 5. Scenario of CME from within an accretion disk corona in 3C147, with asymmetric jets and helical magnetic field structures. The main jet is perpendicular to the accretion disk, while the CME (northern) is inclined to the disk.

Within the accretion disk corona, magnetic loops are expected to exist since magnetic loops are ubiquitous to all cosmic plasmas. Due to differential rotation of the disk, these loops will be pressed together, forming current sheets, and providing reconnection of field lines that eventually will free the magnetic energy from the corona. In case one of the interacting magnetic loops lose magnetic equilibrium, or when large coronal magnetic arcade overlaying the prominence (filament) goes unstable, part of the magnetic structure will be ejected away. The ejected magnetic structure (CME) will have an appearance of a section of the coronal arcade nearly perpendicular to the direction of ejection, as on the sun, and could give rise to the observed SE feature in 3C147. Since the magnetic field structure is generally not symmetric about the accretion disk, then the ejection may not be axial, as is observed in 3C 147 .

Also the origin of the isotropically distributed BLR clouds in AGN may be explained by the existence of many variously sized CMEs (or ejection remnants of CME, with no magnetic fields) ejected in different directions from the accretion disk around the central black hole.

Thus CME with asymmetric jets can explain many features of the radio structure in 3C147, though we expect that some degree of Doppler boosting is also occurring.

With this CME Model we also successfully explained the long disputed puzzle of the exceptional violent variability in intensity, polarization percentage, polarization angle, and jet direction of the well-known BL Lacertae object 0235+164. (Chu et al. 1996)

Recently we find strong evidence that large scale magnetic fields are being ejected away from the nuclear region of 3C147 in the South-East direction and in the North direction. An earlier 2cm polarization map (van Breugel et al. 1984, Fig.4 ) indeed well shows the ejected magnetic fields, but in earlier time, it was natural that these fine details were not noticed and apprehended. As shown in this map, the magnetic field directions, being perpendicular to the polarization E vectors, tend to align with the jet direction in the North and South West. 
While originally during ejection, the ejected magnetic arcade is perpendicular to the jet direction. This is as expected.

These indicate that our previous explanation of the multi-jets in QSR by CME is correct (Chu et al. 1992). we depict now the whole scenario in fig.5. Thus CME with asymmetric jets can explain many features of the radio structure in $3 \mathrm{C} 147$, and probably can explain many phenomena, including the birth of new components, and much of the morphological diversity in other AGN (e.g. Chu et al. 1996).

\section{4. conclusions}

(1) The unusual 3-jet structure found and confirmed in QSR 3C 147 has led to the discovery of helical magnetic fields in AGN \& to the discovery CME and ejection of large scale magnetic fields from AGN.

(2) The helical magnetic field is responsible for the collimation of the jet (through Pinch Effect), and may be of crucial importance for extraction of black hole rotational energy.

(3) The CMEs are responsible for sporadic ejection of jet components in AGN, while the general accepted 2-sided jets pertain to the Quiescent jets.

(4) Observations show that CME with asymmetric jets can explain many features of the radio structure (may not be visible in optical, IR, and $x-, \gamma-$ rays wavelengths) in $3 \mathrm{C} 147$, and probably can explain many phenomena (including the birth of new components) and much of the morphological diversity in many other AGNs ( e.g. Chu et al. 1996), since accretion disk corona is likely to form in other AGNs as well. Acknowledgment: We thank Dr. Li Xiang-Dong for kind help in editing this paper.

\section{References}

Chu, H.S. et al. 1992, in Sub-arcsecond Radio Astronomy, eds. R. J. Davis \& R. S. Booth (Cambridge: Cambridge University Press), 249

Chu, H.S., Baath, L.B., Rantakyro, F.T., Zhang, F.J., \& Nicholson, G. 1996, A\&A, 307, 15

Chu H. S., et al. 1998, in IAU Colloquium 164, Radio Emission from Galactic and Extragalactic Compact Sources, eds. J. A. Zensus, G. B. Taylor, \& J.M. Wrobel, 121

Parker, B.N., 1990, in Galactic and Intergalactic Magnetic Fields, IAU Symp. 140, eds. R.Beck et al., 169

van Breugel, W., Miley, G., \& Heckman, T. 1984 , AJ, 89, 5

Zhang, F.J. et al. 1991, MNRAS, 250, 650 